\title{
La physiothérapie et la sécurité des patients
}

\section{Pia Fankhauser}

Vice-présidente physioswiss, responsable domaine de la communication

Il est intéressant de noter que la recherche des mots «sécurité des patients» ne donne aucun résultat dans la LAMal. C'est donc aux fournisseurs de prestations qu'il revient de la garantir au moyen d'une assurance qualité. physioswiss, l'Association suisse de physiothérapie, est membre du conseil d'administration de la Fondation pour la Sécurité des patients Suisse. Cette dernière organise une semaine d'action qui offre la possibilité de montrer les mesures entreprises par la physiothérapie afin d'assurer la sécurité des patients. Selon l'art. 35 al. 2 litt. e de la LAMal, les physiothérapeutes font partie des "personnes prodiguant des soins sur prescription ou sur mandat médical», l'ancien terme «personnel paramédical» ayant disparu. Du point de vue du patient, c'est l'art. 41 al. 1 de la LAMal qui s'applique: «En cas de traitement ambulatoire, l'assuré a le libre choix entre les fournisseurs de prestations admis et aptes à traiter sa maladie. L'assureur prend en charge les coûts jusqu'à concurrence du tarif applicable au lieu de résidence ou de travail de l'assuré ou dans les environs.» physioswiss a pour mission de promouvoir mais également d'exiger des prestations physiothérapeutiques de qualité. Cette exigence qualitative conditionne une entente commune des professions de la santé sur la réciprocité dans le domaine des soins médicaux ainsi que concernant la mission commune de l'assurance qualité. Ces principes sont fixés dans les statuts de physioswiss.

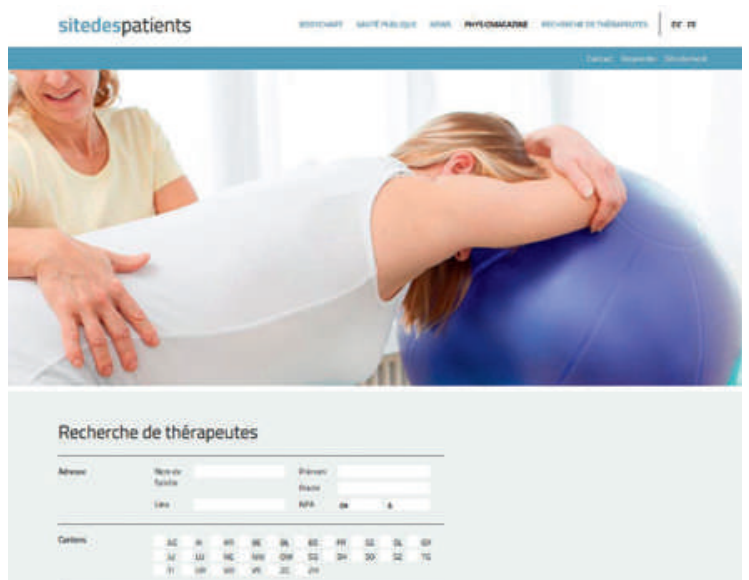

physioswiss offre des informations aux patients ainsi que la possibilité d'effectuer une recherche de thérapeutes.
Déjà lors de la consultation relative à la loi sur les professions de la santé, physioswiss s'exprimait ainsi:

«La création d'un registre professionnel national est une priorité absolue pour physioswiss! Pour la sécurité des patients ainsi que pour la collaboration interdisciplinaire, il est indispensable pour les professions de santé non universitaires de disposer d'un registre professionnel transparent et consultable à tout moment. Etant donné la mobilité croissante des acteurs de la santé, seule une banque de données fiable mise à la disposition des fournisseurs de prestations de la santé au niveau national permettra d'assurer la sécurité des patients ainsi que la collaboration interdisciplinaire.»

Un traitement de physiothérapie commence le plus souvent sur prescription d'un médecin. Ce moyen de communication devrait contenir toutes les données pertinentes pour un traitement rapide et adéquat. Outre le diagnostic principal et les diagnostics secondaires, les principaux médicaments pris par le patient devraient également y figurer. L’anamnèse physiothérapeutique et la pose d'un diagnostic constituent la base du traitement selon le principe du clinical reasoning. Fixer des objectifs sur la base d'un axe temporel permet un contrôle qualitatif par les deux parties et offre la possibilité de signaler d'éventuels problèmes à temps. Le traitement se conclut par un rapport au médecin prescripteur.

Pour les personnes multimorbides et souffrant de maladies chroniques, un réseautage des différents soignants est indispensable. Il s'agit donc d'établir une collaboration interprofessionnelle doublée d'une communication correcte et efficace.

Des technologies modernes existent pour cela (mot-clé: eHealth). Elles doivent toutefois également faire leurs preuves tout en facilitant un échange d'informations pertinentes (!). Nous plaidons pour une révision de la fonction de la prescription et de la réalisation des rapports lors de l'introduction du dossier électronique du patient. Cela est élémentaire en ce qui concerne les soins appropriés et de haute qualité prévus par l'art. 43 al. 6 de la LAMal. Par conséquent, l'informatique représente 
une partie importante de la sécurité des patients. Par ailleurs, elle comprend aussi la protection des données. Le dossier des patients électronique requiert également une collaboration entre les diverses professions ainsi qu'une définition claire des informations nécessaires pour assurer des soins de haute qualité. A cet effet, physioswiss s'est également engagée pour les rapports de la cybermédication et pour le rapport électronique de sortie dans le cadre l'IPAG EPD (le Groupe de travail interprofessionnel pour le dossier électronique du patient).

\section{Il s'agit donc d'établir une collaboration interprofessionnelle doublée d'une communication correcte et efficace.}

C'est peut-être parce que l'ère du patient empowerment les amène à choisir eux-mêmes la thérapie qui leur convient ou parce qu'ils ont été informés que leurs maux peuvent y être traités. Quoi qu'il en soit, les patients se tournent de plus en plus souvent directement vers la physiothérapie. En ce qui concerne les maux de dos non spécifiques, la physiothérapie permet aussi bien d'éviter des examens inutiles que de faciliter une reprise rapide du travail. Pour l'instant, cette pratique est encore réservée aux patients qui paient eux-mêmes les prestations demandées mais il s'agit là d'un processus en pleine évolution. De nouveaux rôles se développent en effet du côté des physiothérapeutes, une évolution qui suit le développement académique de la profession. Le cursus MSc s'est établi de même que le titre de spécialiste clinique physioswiss qui peut être obtenu dans six domaines (pédiatrie, organes internes et vaisseaux, neuromotricité et neurosensibilité, musculo-squelettique, gériatrie, physiothérapie générale, y compris psychomotricité). Ces changements assurent l'évolution de la profession et donc sa qualité. La recherche contribue également à faire bénéficier les patients du traitement qui leur convient.

En 2009, le Swiss Medical Board indiquait que la physiothérapie devait constituer la première option lors de déchirures des ligaments croisés et qu'une opération ne devait être envisagée qu'en cas de graves blessures collatérales ou après un traitement conservateur inefficace. Cette recommandation a été répétée en 2013 après avoir été confirmée par une revue [1]. Les rôles des soins médicaux doivent être adaptés à ces conclusions scientifiques. Une bonne formation et les formations continues permettent d'identifier des «balises rouges» en pratique et assurent ainsi un haut niveau de qualité. physioswiss et les hautes écoles spécialisées s'engagent ensemble pour toutes ces raisons. physioswiss accorde un degré de priorité élevé à la mise en réseau des différents groupes professionnels du système de la santé publique. La Plateforme Interprofessionnalité (www.interprofessionnalite.ch) lancée par Médecins de famille Suisse en est un bon exemple de même que le projet de prévention des chutes du bpa qui a permis l'élaboration de diverses brochures de manière interprofessionnelle. Les soins intégrés de haute qualité sont uniquement possibles lorsque les délimitations professionnelles ne sont plus des murs pour les patients mais que le savoir des compétences constitue la base d'un échange professionnel spécialisé. Cela vaut évidemment aussi bien pour le domaine stationnaire que pour le domaine ambulatoire.

Comment les patients peuvent-ils trouver les informations pertinentes qui les concernent dans un système de santé publique qui se développe de manière aussi dynamique? Comment la qualité peut-elle être rendue visible?

physioswiss offre des informations aux patients (potentiels) ainsi que la possibilité d'effectuer une recherche de thérapeutes parmi ses membres sur le site www.patientenseite.ch. Cette page s'accompagne du physiomagazine. Avec un tirage à 30000 exemplaires, ce journal disponible dans les salles d'attente et dans les hôpitaux traite en allemand et en français de divers sujets de physiothérapie. Le numéro actuel est par exemple consacré aux attaques cérébrales et à ses premiers symptômes.

physiofacts nous permet de nous adresser aux membres du Parlement suisse. Dans le cadre de la semaine d'action de la Fondation pour la Sécurité des patients, nous utilisons ce moyen de communication pour attirer l'attention sur le fait qu'en allemand le terme "traitement" (Behandlung) comporte le mot «main» (Hand) en offrant un désinfectant pour les mains. Nous sommes en effet conscients que seule une collaboration entre tous les groupes professionnels et leurs membres permettra de garantir la sécurité des patients. Nous espérons obtenir ainsi un soutien politique également en ce qui concerne les aspects financiers. En effet, les exigences justifiées de qualité et de savoir ne sont pas gratuites, même dans le domaine de la physiothérapie. La sécurité des patients nous tient réellement à cœur. Elle devrait être une priorité pour chacun.

La sécurité des patients dépend de la mise en réseau de tous les professionnels de la santé ainsi que d'une communication et d'une formation de qualité. Or, tout cela a un prix.

1 Swiss Medical Board. Review Fachbericht «Rupture du LCA: traitement chirurgical ou conservateur?». Zollikon: Swiss Medical Board; 2013. 